\title{
Mortalidad por neumonía en España
}

Pneumonia mortality in Spain

\author{
Enrique Gea-Izquierdo ${ }^{1,2,3^{*}}$
}

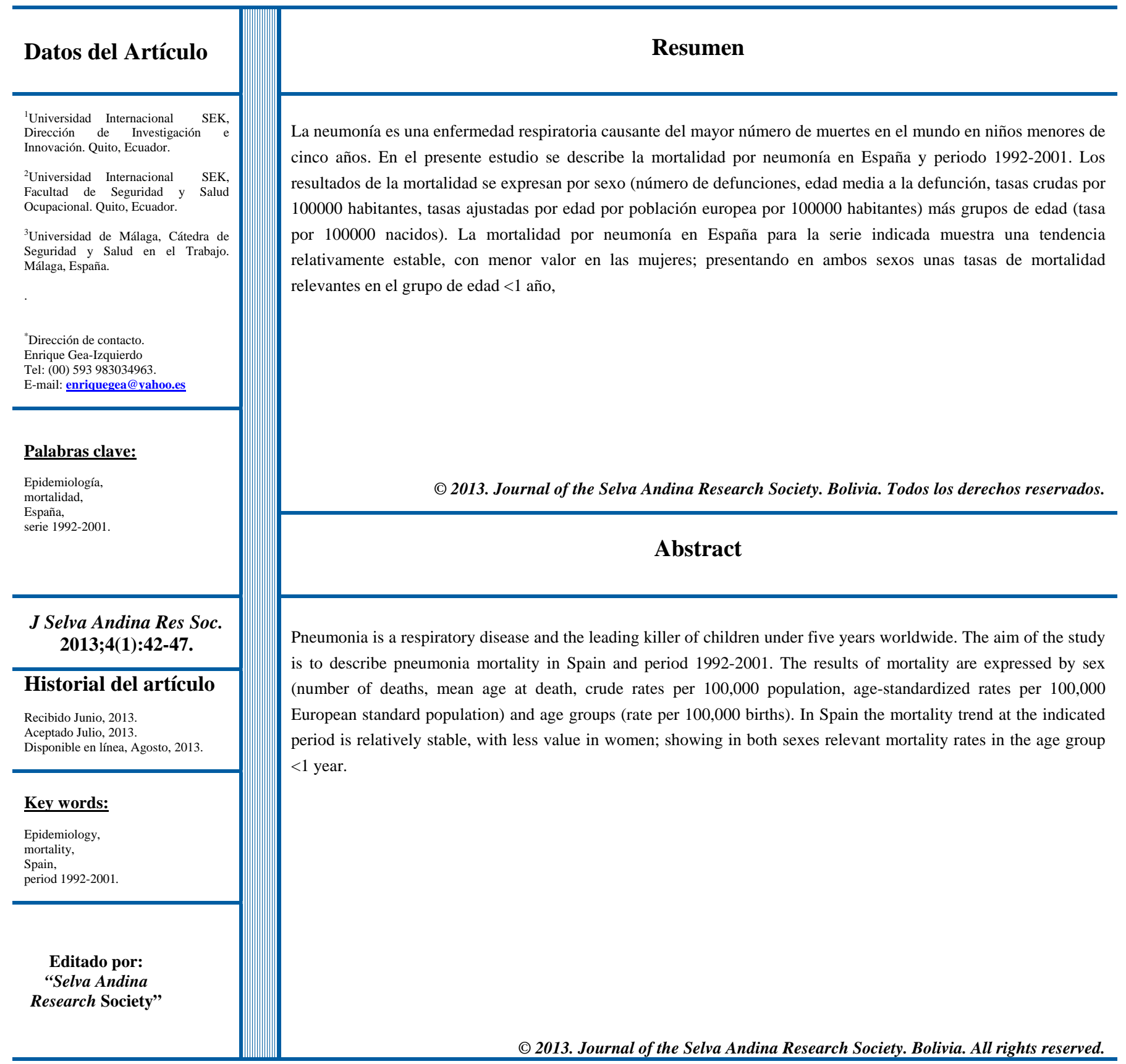


La neumonía es la principal causa individual de mortalidad infantil en todo el mundo, superando en niños menores de cinco años al total de muertes por el sida, malaria y sarampión (UNICEF \& WHO 2006). Diversos autores han descrito esta mortalidad en el mundo (Garenne et al 1992, World Bank 1993, Williams et al 2002, Bryce et al 2005) a pesar de la dificultad para realizar las estimaciones y las diferencias entre el mundo desarrollado y no desarrollado, calculando que en la actualidad hay más de 2 millones de muertes cada año en niños menores de 5 años (Morris et al 2003). Sin embargo, la distribución de la mortalidad para la enfermedad es desigual (United Nations 2013), sobresaliendo África con un $45 \%$ de las muertes totales atribuibles para esta enfermedad (WHO 2013) contrastando con una baja tasa de mortalidad en países desarrollados ( $<1$ por 1000 por año) (Jokinen et al 1993). Por el contrario, las tasas de mortalidad en niños en la mayoría de los países en desarrollo varía entre 60 a 100 por 1000 nacidos vivos y una quinta parte de estas muertes se debe a la neumonía (UNICEF \& WHO 2006). Por lo tanto, la neumonía provoca aproximadamente un 19\% de todas las muertes acontecidas en el mundo en niños menores de cinco años (Rudan et al 2008, Bryce et al 2005), estando fuertemente vinculada a la malnutrición, falta de acceso a cuidados sanitarios y pobreza (WHO \& UNICEF 2009).

La neumonía adquirida en la comunidad (NAC) constituye una causa importante de morbilidad y mortalidad (Gea-Izquierdo 2012), infectando en Estados Unidos cada año de forma alcista a cuatro millones de personas, de las cuales el 20\% requiere hospitalización posterior. Consecuentemente, es un gran contribuidor al coste y consumo de recursos para el cuidado de la salud. Cuando la etiología de la enfermedad no es identificada en los pacientes, el tratamiento es a menudo empírico, propuesto para los agentes biológicos más comunes. Para el tratamiento de la NAC existen una variedad de agentes farmacéuticos, siendo el más notable la cefalosporina y los derivados de la penicilina, los antibacterianos macrólidos/ azálidos, las nuevas tetraciclinas y las más recientes floroquinolonas "respiratorias". La elección de un medicamento normalmente se relaciona con la conformidad del paciente, el perfil de acontecimientos adversos y la presencia de resistencia. De estos hoy la resistencia parece ser el principal factor y Streptococcus pneumoniae la causa más común de NAC (Kuti et al 2002). Aunque este patógeno permanece como la bacteria más comúnmente implicada en este tipo de neumonía otros patógenos respiratorios atípicos han sido aislados, con un aumento en la frecuencia (Gleason 2002); destacando igualmente Haemophilus influenzae tipo b, ciertos virus y el hongo Pneumocystis jiroveci (Calderón Sandubete et al 2011). Destacar que la presentación atípica clínica de pacientes con NAC fue inicialmente reconocida y documentada hace cincuenta años, siendo eventualmente la causa de neumonía en este grupo Mycoplasma pneumoniae y más recientemente Chlamydia pneumoniae. Debido al patrón de susceptibilidad antimicrobiano Legionella ha sido agrupado conjuntamente con las anteriores, presentando un mayor cuadro clínico agudo (Plouffe 2000).

La neumonía es de entre las infecciones nosocomiales la segunda más común y suele prevalecer en los pacientes que están ventilados mecánicamente. La neumonía nosocomial contribuye de manera destacada a la mortalidad de los pacientes, aconteciendo aproximadamente en el $50 \%$ de las muertes por infecciones adquiridas en 
los hospitales. Varios son los factores que sitúan a los pacientes en riesgo para el desarrollo de una neumonía nosocomial (Shorr et al 2013), incluyendo una estancia hospitalaria de larga duración y epidemiología local. Ciertos estudios han demostrado que la iniciación de terapias empíricas apropiadas es esencial en la mejora de los resultados de los pacientes y la reducción de la mortalidad (Baughman et al 1999). Tal y como se ha comentado y a pesar de la existencia de distintos agentes biológicos potencialmente causantes de una neumonía, es cierto que el conocimiento de las estadísticas de mortalidad correspondientes a dicha enfermedad nos puede dar una idea de la repercusión que tiene en la sociedad en términos de salud.

En España el estudio de las defunciones por neumonía ubica la importancia de este tipo de causa de muerte y su relevancia en comparación con otras enfermedades; ocupando el puesto octavo (hombres) y noveno (mujeres) en el orden de causa de mortalidad más relevante para la serie 1992-2001 y grupo de edad >85 años. En la serie las defunciones se encuentran en el intervalo aproximado 3170-4260, presentando valores menores en la mujer con respecto al hombre (tabla 1). La edad media a la defunción por neumonía es aproximadamente 76-80 años para los hombres y 82-85 años para las mujeres (tabla 2), con lo que se observa la diferencia de intervalo de edad media a la defunción y mayor edad de las mujeres.
Tabla 1 Mortalidad por neumonía y sexo (España, 1992-2001. Defunciones)

\begin{tabular}{ccc}
\hline Años & Hombres & Mujeres \\
\hline 1992 & 3320 & 3174 \\
1993 & 3645 & 3314 \\
1994 & 3453 & 3175 \\
1995 & 3729 & 3346 \\
1996 & 3716 & 3396 \\
1997 & 3831 & 3464 \\
1998 & 4102 & 3728 \\
1999 & 4254 & 3998 \\
2000 & 3877 & 3679 \\
2001 & 3791 & 3260 \\
\hline
\end{tabular}

Tabla 2 Mortalidad por neumonía y sexo (España, 1992-2001. Edad media a la defunción)

\begin{tabular}{ccc}
\hline Años & Hombres & Mujeres \\
\hline 1992 & 76,67 & 82,33 \\
1993 & 76,84 & 83,04 \\
1994 & 77,32 & 83,51 \\
1995 & 77,82 & 83,57 \\
1996 & 78,07 & 83,82 \\
1997 & 78,18 & 83,55 \\
1998 & 79,49 & 84,1 \\
1999 & 79,09 & 84,31 \\
2000 & 79,55 & 84,17 \\
2001 & 79,64 & 84,93 \\
\hline
\end{tabular}

En la tabla 3 se presenta la tasa cruda en España por 100000 habitantes para la mortalidad por neumonía según sexo y serie estudiada, siendo sensiblemente menor en mujeres que en hombres y con aumento a lo largo de los años. En cambio en la representación de la tasa ajustada por edad por población europea por 100000 habitantes (tabla 4) se produce una disminución hacia el final de la serie aún con valores menores en el caso de las mujeres. 
Tabla 3 Mortalidad por neumonía y sexo

(España, 1992-2001.

Tasa cruda por 100000 habitantes)

\begin{tabular}{ccc}
\hline Años & Hombres & Mujeres \\
\hline 1992 & 17,38 & 15,94 \\
1993 & 19,05 & 16,61 \\
1994 & 18,02 & 15,89 \\
1995 & 19,43 & 16,71 \\
1996 & 19,34 & 16,93 \\
1997 & 19,92 & 17,24 \\
1998 & 21,31 & 18,53 \\
1999 & 21,95 & 19,75 \\
2000 & 19,84 & 18,04 \\
2001 & 19,23 & 15,86 \\
\hline
\end{tabular}

Es fácil identificar el aumento de la tasa de mortalidad en hombres por neumonía según se incrementa el grupo de edad a partir de 25-34 años (tabla 5), cobrando mucha importancia para individuos por encima de 85 años y con bastante relevancia en el intervalo 75-84. Al mismo tiempo se puede observar como para las tasas $<1$ año se presentan años reseñables (1993 y 1992) considerando el resto de intervalos con una significación menor. En las mujeres también es importante la tasa para <1 año (tabla 6), especialmente los años 1992, 1993 y 1995. El intervalo 45-54 se podría asemejar al 25-34 de los hombres, con aumento de las tasas según la edad, aunque de nuevo hay que destacar la tasa menor de las mujeres con respecto a los hombres.

Tabla 4 Mortalidad por neumonía y sexo (España, 1992-2001.

Tasa ajustada por edad por población europea por 100000 habitantes)

\begin{tabular}{ccc}
\hline Años & Hombres & Mujeres \\
\hline 1992 & 17,52 & 9,74 \\
1993 & 18,84 & 9,80 \\
1994 & 17,3 & 9,04 \\
1995 & 18,23 & 9,27 \\
1996 & 17,73 & 9,08 \\
1997 & 17,9 & 9,05 \\
1998 & 18,76 & 9,47 \\
1999 & 18,72 & 9,78 \\
2000 & 16,69 & 8,77 \\
2001 & 15,97 & 7,45 \\
\hline
\end{tabular}

Tabla 5 Mortalidad en hombres por neumonía y grupo de edad (España, 1992-2001. Tasa por 100000 nacidos)

\begin{tabular}{|c|c|c|c|c|c|c|c|c|c|c|c|}
\hline \multirow{2}{*}{ Años } & \multicolumn{11}{|c|}{ Grupo de edad (años) } \\
\hline & $<1$ & $0-4$ & $5-14$ & $15-24$ & $25-34$ & $35-44$ & $45-54$ & $55-64$ & $65-74$ & $75-84$ & $>85$ \\
\hline 1992 & 6,84 & 1,47 & 0,19 & 0,39 & 2,50 & 2,79 & 4,90 & 11,88 & 37,42 & 176,05 & 692,50 \\
\hline 1993 & 10,53 & 2,67 & 0,35 & 0,77 & 2,37 & 2,66 & 5,05 & 12,21 & 39,52 & 185,55 & 765,98 \\
\hline 1994 & 4,71 & 1,2 & 0,33 & 0,33 & 2,31 & 3,15 & 4,17 & 10,94 & 36,74 & 175,49 & 702,88 \\
\hline 1995 & 3,20 & 1,00 & 0,17 & 0,58 & 2,44 & 2,68 & 4,34 & 10,83 & 38,39 & 186,67 & 751,97 \\
\hline 1996 & 3,21 & 1,20 & 0,09 & 0,59 & 1,73 & 2,52 & 4,21 & 12,24 & 38,92 & 176,42 & 726,04 \\
\hline 1997 & 4,73 & 1,11 & 0,23 & 0,47 & 1,07 & 3,04 & 4,67 & 11,60 & 43,23 & 167,43 & 737,31 \\
\hline 1998 & 4,23 & 0,91 & 0,19 & 0,29 & 0,88 & 2,02 & 4,36 & 10,83 & 42,09 & 178,62 & 826,5 \\
\hline 1999 & 3,58 & 0,95 & 0,05 & 0,46 & 1,16 & 2,59 & 4,42 & 10,69 & 42,28 & 179,58 & 803,95 \\
\hline 2000 & 3,40 & 0,94 & 0,19 & 0,20 & 0,50 & 2,37 & 3,85 & 10,02 & 34,36 & 162,63 & 733,26 \\
\hline 2001 & 0,48 & 0,20 & 0,05 & 0,28 & 0,81 & 1,98 & 4,68 & 9,73 & 32,78 & 148,41 & 717,88 \\
\hline
\end{tabular}


Tabla 6 Mortalidad en mujeres por neumonía y grupo de edad (España, 1992-2001. Tasa por 100000 nacidos)

\begin{tabular}{|c|c|c|c|c|c|c|c|c|c|c|c|}
\hline \multirow{2}{*}{ Años } & \multicolumn{11}{|c|}{ Grupo de edad (años) } \\
\hline & $<1$ & $0-4$ & $5-14$ & $15-24$ & $25-34$ & $35-44$ & $45-54$ & $55-64$ & $65-74$ & $75-84$ & $>85$ \\
\hline 1992 & 10,94 & 2,60 & 0,32 & 0,44 & 0,99 & 0,99 & 1,07 & 3,84 & 15,98 & 94,27 & 489,60 \\
\hline 1993 & 6,97 & 1,78 & 0,08 & 0,28 & 0,82 & 0,71 & 1,14 & 4,33 & 15,48 & 96,68 & 504,81 \\
\hline 1994 & 4,47 & 1,38 & 0,04 & 0,44 & 0,52 & 0,89 & 1,34 & 2,93 & 13,87 & 93,39 & 466,49 \\
\hline 1995 & 6,25 & 1,60 & 0,09 & 0,32 & 0,61 & 1,02 & 1,05 & 3,77 & 13,23 & 92,39 & 487,73 \\
\hline 1996 & 2,84 & 0,86 & 0,33 & 0,35 & 0,57 & 0,96 & 0,95 & 3,79 & 13,33 & 91,75 & 475,89 \\
\hline 1997 & 3,35 & 1,08 & 0,24 & 0,26 & 0,69 & 0,76 & 1,61 & 3,31 & 14,66 & 93,67 & 453,05 \\
\hline 1998 & 3,41 & 0,87 & 0,25 & 0,27 & 0,53 & 0,78 & 1,72 & 3,96 & 14,87 & 91,11 & 494,52 \\
\hline 1999 & 1,63 & 0,34 & 0,35 & 0,14 & 0,62 & 0,69 & 1,53 & 3,92 & 16,51 & 92,41 & 516,63 \\
\hline 2000 & 3,12 & 0,89 & 0,10 & 0,18 & 0,55 & 1,11 & 1,26 & 3,08 & 14,36 & 82,91 & 461,19 \\
\hline 2001 & 0,51 & 0,43 & 0,05 & 0,22 & 0,42 & 0,69 & 1,20 & 2,19 & 10,93 & 69,68 & 412,99 \\
\hline
\end{tabular}

Tabla 7 Mortalidad en ambos sexos por neumonía y edad menor a 1 año (España, 1992-2001. Tasa por 100000 nacidos)

\begin{tabular}{cc}
\hline & \multicolumn{1}{c}{$\begin{array}{l}\text { Grupo } \\
\text { (años) }\end{array}$} \\
\cline { 2 - 2 } Años & $<1$ \\
\cline { 2 - 2 } 1992 & 8,82 \\
1993 & 8,81 \\
1994 & 4,59 \\
1995 & 4,68 \\
1996 & 3,03 \\
1997 & 4,06 \\
1998 & 3,83 \\
1999 & 2,63 \\
2000 & 3,27 \\
2001 & 0,49 \\
\hline
\end{tabular}

Según se ha resaltado, en España el grupo de edad < 1 año presenta interés para la mortalidad por neumonía. Considerando las tablas 5 y 6 se ha estimado conveniente representar la mortalidad en ambos sexos (tabla 7), en donde se aprecia con mayor claridad el alcance de la mortalidad en esa franja de edad.
En España y para la serie 1992-2001 las defunciones por neumonía, año y sexo se aproximan bastante, con valores menores en las mujeres y con una edad media a la defunción mayor en éstas últimas con respecto a los hombres. Las tasas crudas por 100000 habitantes son bastantes similares en ambos sexos, mientras que en hombres las tasas ajustadas por edad por población europea por 100000 habitantes prácticamente duplican a las de las mujeres. Por último, destacar que atendiendo a la mortalidad por neumonía, sexo y grupos de edad, es en edades avanzadas donde se presentan los mayores valores; aun apreciando en ambos sexos la mortalidad en edad menor a un año, en la que para la serie descrita se produce cronológicamente un descenso aproximado en las tasas por 100000 nacidos.

\section{Conflictos de interés}

El presente trabajo no genera conflictos de interés. 


\section{Literatura citada}

Baughman RP, Tapson V, Mc. Ivor A. The diagnosis and treatment challenges in nosocomial pneumonia. Diagn Microbiol Infect. Dis. 1999;33(2):131-139.

Bryce J, Boschi-Pinto C, Shibuya K, Black RE; WHO Child Health and Epidemiology Research Group. WHO estimates of the causes of death in children. Lancet. 2005;365:1147-1152.

Calderón Sandubete E, De Armas Rodríguez Y, Capó de Paz V. Pneumocystis jirovecii: Cien años de historia. Rev Cubana Med Trop. 2011;63(2):97-116.

Garenne M, Ronsmans C, Campbell H. The magnitude of mortality from acute respiratory infections in children under 5 years in developing countries. World Health Stat Q. 1992;45:180-191.

Gea-Izquierdo E. Epidemiología y prevención de las enfermedades respiratorias. Quito: Universidad Internacional SEK-Ecuador; 2012.

Gleason PP. The emerging role of atypical pathogens in community-acquired pneumonia. Pharmacotherapy. 2002;22(1):2S-11S.

Jokinen C, Heiskanen L, Juvonen H, Kallinen S, Karkola K, Korppi M, et al. Incidence of community-acquired pneumonia in the population of four municipalities in eastern Finland. Am J Epidemiol. 1993;137(9):977-988.

Kuti JL, Capitano B, Nicolau DP. Cost-effective approaches to the treatment of communityacquired pneumonia in the era of resistance. Pharmacoeconomics. 2002;20(8):513-528.

Morris SS, Black RE, Tomaskovic L. Predicting the distribution of under-five deaths by cause in countries without adequate vital registration systems. Int J Epidemiol. 2003;32(6):1041-1051.
Plouffe JF. Importance of atypical pathogens of community-acquired pneumonia. Clin Infect Dis. 2000;31(2):35-39.

Rudan I, Boschi-Pinto C, Biloglav Z, Mulholland K, Campbell H. Epidemiology and etiology of childhood pneumonia. Bull World Health Organ. 2008;86(5):408-416.

Shorr AF, Zilberberg MD, Reichley R, Kan J, Hoban A, Hoffman J, et al. Readmission following hospitalization for pneumonia: The impact of pneumonia type and its implication for hospitals. Clin Infect Dis. 2013.

UNICEF/WHO. Pneumonia: The forgotten killer of children. UNICEF; 2006.

United Nations. United Nations Millennium Development Goals. Disponible en: http://www.un.org/apps/news/story.asp?NewsID $=44639$ [Accesible el 3 de junio de 2013].

WHO. World Health statistics. Geneva: WHO; $2007 . \quad$ Disponible en: http://www.who.int/whosis/whostat2007.pdf [Accesible el 3 de junio de 2013].

WHO/UNICEF. Global action plan for prevention and control of pneumonia (GAPP). WHO/UNICEF; 2009.

Williams BG, Gouws E, Boschi-Pinto C, Bryce J, Dye C. Estimates of world-wide distribution of child deaths from acute respiratory infections. Lancet Infect Dis. 2002;2:25-32.

World Bank. World development report 1993: Investing in health. Washington, DC: Oxford University Press; 1993. 